\title{
Self-pulsation in Raman fiber amplifiers
}

\section{Pedersen, Martin Erland Vestergaard; Ott, Johan Raunkjær; Rottwitt, Karsten}

\section{Published in:}

Proceedings of the 11th International Conference on Transparent Optical Networks. Azores, Portugal, 2009

Link to article, DOI:

10.1109/ICTON.2009.5185179

Publication date:

2009

Document Version

Publisher's PDF, also known as Version of record

Link back to DTU Orbit

Citation (APA):

Pedersen, M. E. V., Ott, J. R., \& Rottwitt, K. (2009). Self-pulsation in Raman fiber amplifiers. In Proceedings of the 11th International Conference on Transparent Optical Networks. Azores, Portugal, 2009 (pp. 1-2). IEEE. https://doi.org/10.1109/ICTON.2009.5185179

\section{General rights}

Copyright and moral rights for the publications made accessible in the public portal are retained by the authors and/or other copyright owners and it is a condition of accessing publications that users recognise and abide by the legal requirements associated with these rights.

- Users may download and print one copy of any publication from the public portal for the purpose of private study or research.

- You may not further distribute the material or use it for any profit-making activity or commercial gain

- You may freely distribute the URL identifying the publication in the public portal 


\title{
Self-Pulsation in Raman Fiber Amplifiers
}

\author{
M. E. V. Pedersen, J. R. Ott, K. Rottwitt \\ Technical University of Denmark (DTU), Department of Photonic Engineering (DTU Fotonik) \\ Build. 345V, DK-2800,Kgs. Lyngby, Denmark \\ martin.pedersen@fotonik.dtu.dk
}

\section{ABSTRACT}

Dynamic behavior caused by Brillouin scattering in Raman fiber amplifiers is studied. Modes of self-pulsation steady state oscillations are found. Their dependence on amplification scheme is demonstrated.

Keywords: self-pulsation, Raman amplified, stimulated Brillouin scattering.

\section{SUMMARY}

When an intense continuous wave $(\mathrm{CW})$ beam propagate through an optical fiber it creates a backward propagating wave due to spontaneous Brillouin scattering. This backward propagating Brillouin shifted wave appears approximately $10 \mathrm{GHz}$ from the launched $\mathrm{CW}$ beam, when a typical silica optical fiber is used. The spontaneous backward propagating wave is amplified due to stimulated Brillouin scattering (SBS), where the input $\mathrm{CW}$ signal act as a pump. As the power of the input $\mathrm{CW}$ signal is increased, the Brillouin gain is increased and consequently the backward propagating power increases to a level where it starts to deplete the input signal. If the input signal power is increased even further, the depletion causes the input signal power to drop, and if at the same time the light is in a cavity, for example due to reflections at the fiber ends, caused by connections of different types of fibers, then the system enters eventually a mode of self-pulsation. The repetition rate of the pulses corresponds to the round trip time of the fiber length and the rise/fall time of the pulses is defined by the strength of the Brillouin scattering and the launched signal power [1]. In the following the impact of Raman amplification on self-pulsation due to SBS is investigated numerically. The oscillation phenomena was numerically investigated by R. V. Johnson and J. H. Marburger in 1971 [1] and analytically in the case with no attenuation nor Raman amplification by I. Bar-Joseph and co-workers [2] and has since then been the subject of much investigation $[3,4,5]$. The work reported here is motivated by the recent development of highly nonlinear fibers, and the interest in distributed and discrete amplifiers such as Raman amplifiers and the recent use of Raman amplification in Brillouin fiber lasers [6].

The setup consist of a single mode inversion symmetric fiber of length $L$ placed between two fiber end reflectors having power reflectivities, $R_{1}$ and $R_{2}$ that reflect the light back into the fiber. In the model the two reflections are assumed identical. For a polarization maintaining single mode fiber, neglecting higher order Brillouin scattering, the governing equations are $[1,7]$

$$
\begin{aligned}
& \frac{1}{v_{g, \mathrm{~S}}} \frac{\partial P_{\mathrm{S}}^{ \pm}}{\partial t} \pm \frac{\partial P_{\mathrm{S}}^{ \pm}}{\partial z}=-g_{\mathrm{B}} P_{\mathrm{S}}^{ \pm} P_{\mathrm{B}}^{\mp}+g_{\mathrm{R}} P_{\mathrm{S}}^{ \pm}\left(P_{\mathrm{R}}^{+}+P_{\mathrm{R}}^{-}\right)-\alpha_{\mathrm{S}} P_{\mathrm{S}}^{ \pm}, \\
& \frac{1}{v_{g, \mathrm{~B}}} \frac{\partial P_{\mathrm{B}}^{ \pm}}{\partial t} \pm \frac{\partial P_{\mathrm{B}}^{ \pm}}{\partial z}=g_{\mathrm{B}} P_{\mathrm{S}}^{\mp} P_{\mathrm{B}}^{ \pm}+g_{\mathrm{R}} P_{\mathrm{B}}^{ \pm}\left(P_{\mathrm{R}}^{+}+P_{\mathrm{R}}^{-}\right)-\alpha_{\mathrm{B}} P_{\mathrm{B}}^{ \pm}, \\
& \frac{1}{v_{g, \mathrm{R}}} \frac{\partial P_{\mathrm{R}}^{ \pm}}{\partial t} \pm \frac{\partial P_{\mathrm{R}}^{ \pm}}{\partial z}=-g_{\mathrm{R}} \frac{\omega_{R}}{\omega_{S}} P_{\mathrm{R}}^{ \pm}\left(P_{\mathrm{S}}^{+}+P_{\mathrm{S}}^{-}+P_{\mathrm{B}}^{+}+P_{\mathrm{B}}^{-}\right)-\alpha_{\mathrm{R}} P_{\mathrm{R}}^{ \pm},
\end{aligned}
$$

where $P_{\mathrm{S}}^{ \pm}=P_{\mathrm{S}}^{ \pm}(z, t), P_{\mathrm{B}}^{ \pm}=P_{\mathrm{B}}^{ \pm}(z, t)$ and $P_{\mathrm{R}}^{ \pm}=P_{\mathrm{R}}^{ \pm}(z, t)$ are the powers of the signal, Brillouin and Raman waves, the $+(-)$ superscripts indicates forward(backward) propagating waves, $g_{i}$ is the Brillouin $(i=\mathrm{B})$ or Raman ( $i=\mathrm{R}$ ) gain coefficient, $z$ is the length, and $t$ is the time. Furthermore $\alpha_{\mathrm{S}}, \alpha_{\mathrm{B}}$, and $\alpha_{\mathrm{R}}$ are the attenuation coefficients which for simplicity are identical. Similar $v_{g, \mathrm{~S}}, v_{g, \mathrm{~B}}$, and $v_{g, \mathrm{R}}$ are the group velocities, which are assumed the same for all waves. $\omega_{S}$ and $\omega_{R}$ are the angular frequencies of the signal wave and the Raman wave respectively. The difference in energy between the Raman wave and the signal and Brillouin waves is included in equation (1) through the term $\omega_{R} / \omega_{S}$. The frequency difference between the signal and Brillouin waves is neglected. The pump for the Raman amplifier is assumed to have a linewidth broad enough to neglect Brillouin scattering on the Raman pump. Both Raman and Brillouin response is assumed instantaneous since we are dealing with $\mathrm{CW}$ and quasi-CW signal only.

Figure 1 shows what level of signal input power that is required to get a transmitted signal that is pulsing for a round trip power reflectivity in the range from 0 to $-140 \mathrm{~dB}$. This is shown for two amplification schemes, a distributed and a discrete counter pumped Raman amplifier (Fig. 1a and Fig. 1b, respectively). The distributed Raman amplifier is a $100 \mathrm{~km}$ long fiber amplifier, with a total loss of $20 \mathrm{~dB}$. The Brillouin and the Raman gain coefficient is $0.14 \mathrm{~W}^{-1} \mathrm{~m}^{-1}$ and $0.7 \mathrm{~W}^{-1} \mathrm{~km}^{-1}$, respectively [8]. In this case (Fig. 1a) three cases are compared, one 
where loss is neglected, one with loss, and one where the loss is counterbalanced by the Raman amplification. The Raman pump power is chosen exactly large enough to provide a Raman gain that equals the intrinsic attenuation. This is done by assuming an undepleted pump with no reflections, and equals $0.3 \mathrm{~W}$. The discrete Raman amplifier, consists of a $200 \mathrm{~m}$ long fiber with an intrinsic attenuation of $0.8 \mathrm{~dB} / \mathrm{km}$ and a Brillouin and a Raman gain coefficient equal to $0.8 \mathrm{~W}^{-1} \mathrm{~m}^{-1}$ and $5 \mathrm{~W}^{-1} \mathrm{~km}^{-1}$, respectively [7]. In Fig. $1 \mathrm{~b}$ three cases are compared, one where loss is neglected, one with loss, and one where the signal is amplified by $20 \mathrm{~dB}$ by the discrete Raman amplifier. As in the case of the distributed amplifier, the Raman pump power is calculated assuming an undepleted pump with no reflections. For a $20 \mathrm{~dB}$ gain the Raman pump power equals $4.7 \mathrm{~W}$.
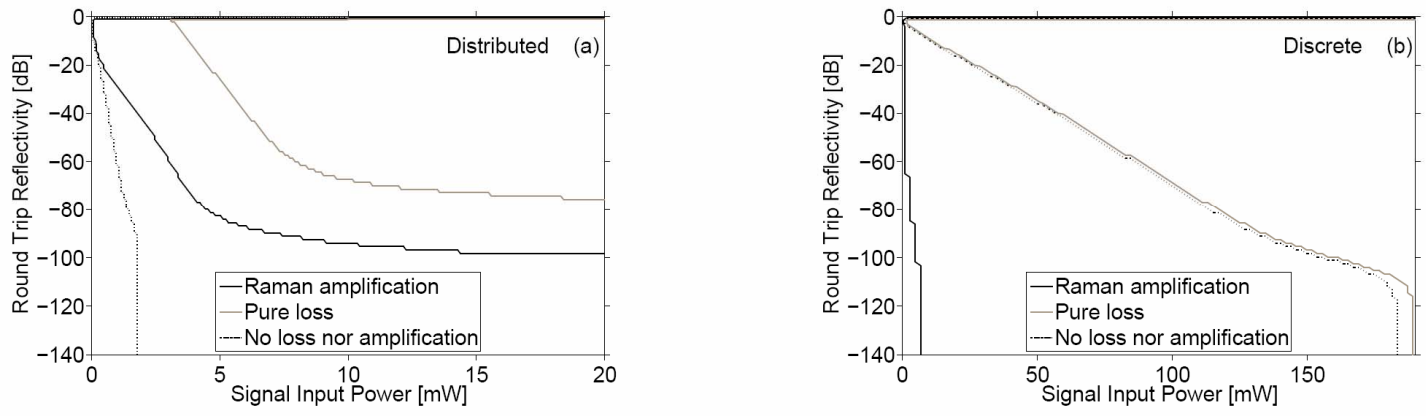

Figure 1. Self-pulsation stability regions. The regions below the curves corresponds to CW output and the regions above corresponds to steady state oscillations. The round trip reflectivity is defined as $10 \log _{10}\left(R_{1} R_{2}\right)$. If both fiber ends are cleaved at a $90^{\circ}$ angle to the direction of the fiber, the reflection at each end equals $\approx-15 \mathrm{~dB}$, corresponding to a round trip reflectivity of $-30 \mathrm{~dB}$ however, typical components and splices have significant lower reflections. The Brillouin is seeded with a single photon CW opposite the input signal.

The plots illustrate that for low signal power levels, high reflections are needed to reach self-pulsation. It is noted in Fig. 1a that the no loss region appears to be cut sharply for an input signal power close to $2 \mathrm{~mW}$, similar in Fig. $1 \mathrm{~b}$ around $180 \mathrm{~mW}$. This is because the Brillouin wave gains so much power in one pass, which causes the system to oscillate. It is observed that the stability for the discrete Raman amplifier cuts off at around $7 \mathrm{~mW}$, while the distributed Raman amplifier remain stable beyond $20 \mathrm{~mW}$. This is believed to be contributed to the depletion of the Raman pump in the distributed amplifier so that the oscillation are inhibited by the fiber loss.

In conclusion, it is demonstrated that Brillouin scattering in optical fiber amplifiers may lead to steady state oscillations, when a launching a CW signal into the amplifier. We have shown that there is a significant difference between using a discrete or distributed Raman amplifier, where the distributed amplifier is significantly more robust against self-pulsation considering a fixed signal power level and a fixed total reflectivity.

\section{REFERENCES}

[1] R. V. Johnson and J. H. Marburger, "Relaxation oscillation in stimulated Raman and Brillouin scattering", Phys. Rev. A 4, 1175-1182 (1971).

[2] I. Bar-Joseph, A. A. Friesem, E. Lichtman and R. G. Waarts, "Steady and relaxation oscillations of stimulated Brillouin scattering in single-mode optical fibers", JOSA B 2, 1606, 1985.

[3] R. G. Harrison, J. S. Uppal, A. Johnstone and J. V. Moloney, "Evidence of chaotic stimulated Brillouin scattering with weak feedback", Phys. Rev. Lett. 65, 167-170 (1990).

[4] A. L. Gaeta and R. W. Boyd, "Stimulated Brillouin scattering in the presence of external feedback", J. Nonlinear Opt. Phys. Mater. 1, 581-594 (1992).

[5] M. Dämmig, G.Zinner, F. Mitschke and H. Welling, "Stimulated Brillouin scattering in fibers with and without external feedback", Phys. Rev. A 48, 3301-3309 (1993).

[6] J. W. Lou, F. K. Fatemi, and M. Currie, "Brillouin fibre laser enhanced by Raman amplification", Elect. Lett. 40, (2004)

[7] K. Rottwitt and A.J. Stentz, "Optical Fiber Telecommunications", (Academic Press, 2002), Chap. 8.

[8] G. P. Agrawal, "Nonlinear Fiber Optics", 3rd ed., (Academic Press 2001), Chap. 8 \& 9. 